\title{
Caracterización de los residuos sólidos en la UNTELS.
}

${ }^{1}$ Beatriz Luisa Salvador Gutiérrez ${ }^{\text {dol }},{ }^{2}$ Margarita Fredesvinda Murillo Manrique,
${ }^{2}$ Myrna Manco Caycho, ${ }^{2}$ Ángela C. Huachua Paucarpura.

${ }^{1}$ Universidad Nacional Mayor de San Marcos. Lim, Perú

${ }^{2}$ Universidad Nacional Tecnológica de Lima Sur, Lima, Perú

Recibido: 05/09/2021 Revisado: 03/10/2021 Aceptado: 16/11/2021 Publicado: 30/01/2022

\section{Resumen}

El Ministerio del Ambiente (MINAM) promueve que las universidades incorporen procesos de gestión ambiental y contribuyan a la solución de la problemática ambiental en el Perú debido a la amplitud de sus funciones de formación profesional, investigación, extensión y gestión, en ese contexto y como parte de la investigación titulada "Implementación de un plan de gestión de residuos sólidos en la UNTELS" nos proponemos caracterizar los residuos sólidos en la UNTELS. Para la recolección de datos se ha utilizado las técnicas de observación y trabajo de campo. Se obtuvieron como resultados globales en la generación per cápita (GPC) de $0.02 \mathrm{Kg}$ y en la generación total $58.22 \mathrm{Kg}$ /día, equivalente a 15.14 ton/año. Se encontró que los desechos sólidos (52\%) ocupan un primer lugar, seguido de materia orgánica (16\%), la generación de desechos sólidos oscila entre 20.71 y 39.57 Kg/día, la generación de residuos electrónicos no es significativa y que el plástico PET constituye el mayor tipo de residuo reaprovechable, seguido por el papel.

Palabras clave: Residuos sólidos, caracterización y composición de residuos sólidos.

\begin{abstract}
.
The Ministry of the Environment (MINAM) encourages universities to incorporate environmental management processes and contribute to the solution of environmental problems in Peru due to the breadth of their professional training, research, extension, and management
\end{abstract}


functions, in this context, the This research aims to implement a solid waste management plan (RRSS) at the National Technological University of Lima Sur (UNTELS).

Based on the regulations and the various methodological guides prepared by MINAM for educational institutions, the following dimensions have been considered: Political-institutional, technical-operational and environmental education. Various techniques such as observation, survey and field work have been used for data collection. Regarding the characterization of the RRSS, overall results were obtained in the per capita generation (GPC) of $0.02 \mathrm{Kg}$ and in the total generation $58.22 \mathrm{Kg} /$ day, equivalent to 15.14 ton / year. It was found that solid waste $(52 \%)$ occupies a first place, followed by organic matter (16\%), the generation of solid waste ranges between 20.71 and $39.57 \mathrm{Kg} /$ day, the generation of electronic waste is not significant and that plastic PET is the largest type of reusable waste, followed by paper.

Keywords: Solid waste, solid waste characterization and composition

\section{Introducción}

El Perú está enfrentando una realidad difícil, pues está generando 18,131 toneladas de basura al día con un inadecuado manejo de residuos sólidos y según un reporte del (Organismo de Evaluación y Fiscalización Ambiental, 2014) determina que la generación de basura per cápita es de $0.65 \mathrm{~kg}$ en nuestra capital. La Universidad Nacional Tecnológica de Lima Sur (UNTELS) ubicada en Villa el Salvador, con una población de 2945 habitantes (Semestre 2017-I) y un área de 23,680 m , no escapa de tal realidad. Lamentablemente se presentan deficiencias en cuanto al proceso de recolección y almacenamiento de residuos sólidos, procesos que requieren de una infraestructura adecuada. Nuestro objetivo fue identificar la cantidad y tipos de residuos sólidos generados por la población universitaria de la UNTELS. Las investigaciones realizadas por

(Ferreira et al., 2017), (Alves Eustáquio et al., 2019), (Vásquez, E., Bermejo, D., Sánchez \& Sarmiento Ramos, 2013), (Ramírez, 2014), (Eche Guerra \& Sánchez Melchor, 2016), el (Comité Ambiental Universitario UNAP, 2018), (Cabañas Pinedo et al., 2019), Amézquita Cadena (2019) encontró dejan en claro que la preocupación por el cuidado del medio ambiente en el ámbito universitario es un desafío que debe ser abordado con responsabilidad. 


\section{Materiales y métodos}

\section{a. Clasificación de la Investigación}

Por el nivel de conocimientos que se adquieren, la presente investigación se clasifica como descriptiva y por la clase de medios utilizados para obtener los datos, es una investigación de campo.

\section{b. Etapas para el desarrollo del Estudio de Caracterización}

El Ministerio del Ambiente (2015a) describe que el estudio de caracterización se realizó para determinar los parámetros: Generación y composición. Se adaptaron las etapas señaladas en la Guía metodológica para el desarrollo del Estudio de Caracterización de Residuos Sólidos Municipales (EC-RSM), el flujograma se observa en la Figura 1. Para determinar los indicadores de generación per cápita de residuos sólidos se usaron métodos de estimación indirectos adaptando de la Guía Metodológica para la elaboración del estudio de caracterización de residuos sólidos (Ministerio del Ambiente, 2015a), pág. 34) y para la generación total, mensual y composición física de residuos sólidos se utilizó el criterio de (Ramírez, 2014), con estos parámetros se podrá dimensionar el equipamiento para la recolección de residuos sólidos, transporte e infraestructura. Se identificaron la cantidad y tipos de residuos que se generan, siguiendo las recomendaciones de (Tello et al., 2010). 


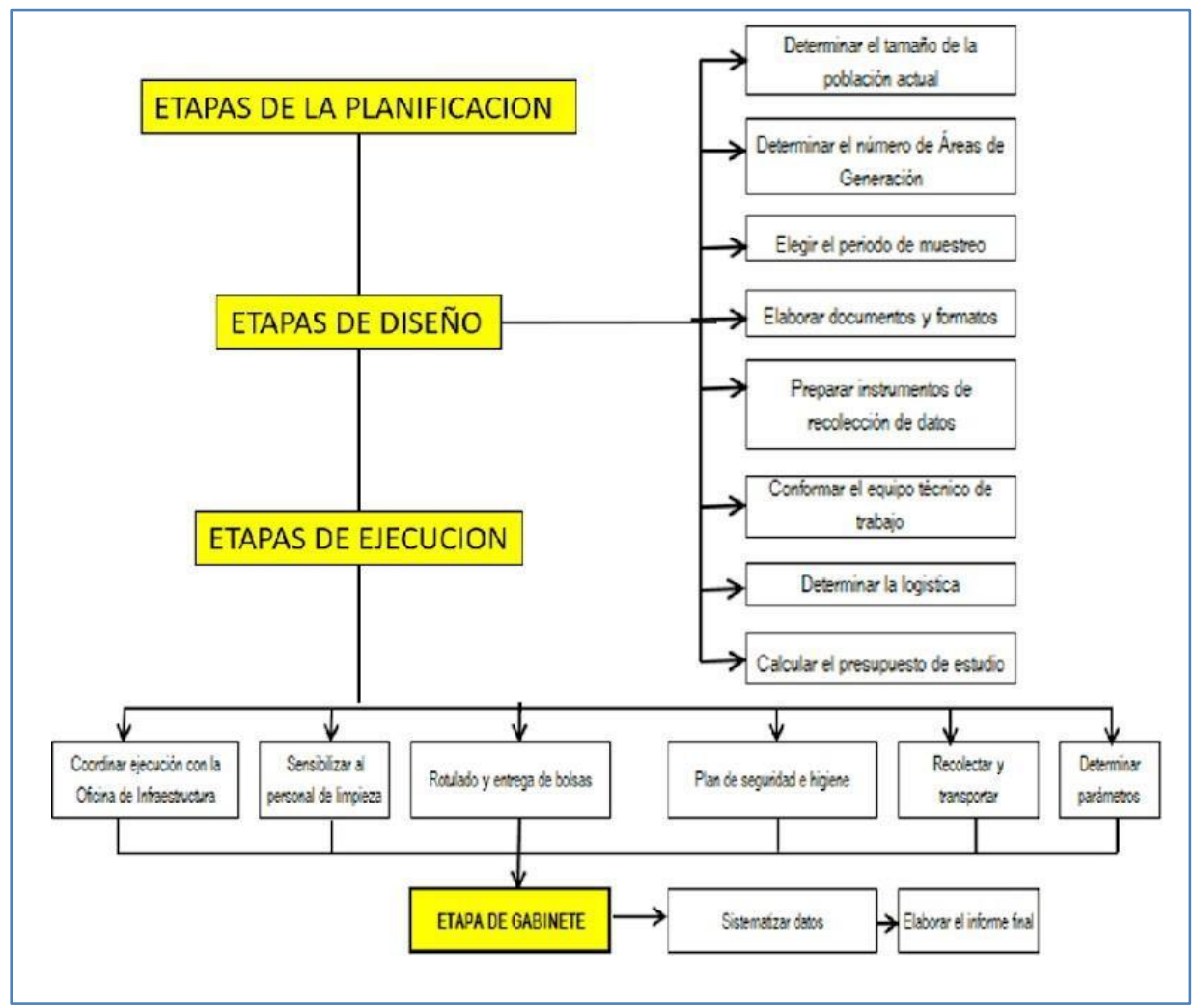

Figura 1: Flujograma de las etapas para el Desarrollo del estudio de caracterización de residuos municipales (ER-RSM) Fuente: Adaptado de Guía metodológica para el desarrollo del estudio de Caracterización de Residuos Sólidos Municipales.

\section{c. Etapa de planificación}

Para planificar el estudio de caracterización el equipo investigador utilizó la técnica de la visualización in situ teniendo como resultado que los trabajadores de limpieza recolectan los residuos de todas las áreas de generación sin separación por tipo de desecho, estos residuos se llevan al lugar de acopio dentro del Campus Universitario y se espera al carro recogedor de basura de la Municipalidad para que pueda recogerlos. En la presente investigación no se han considerado los residuos peligrosos provenientes de los laboratorios y del Área de Salud, ni los residuos del comedor universitario.

\section{d. Etapa de diseño}

La población estuvo constituida por los residuos sólidos producidos por los 2945 integrantes de la comunidad Untelsina (año 2016). La muestra consistió en elegir una semana aleatoriamente dentro del periodo de estudio. La caracterización de los residuos sólidos se realizó para8 días consecutivos midiendo totalmente los residuos producidos utilizando la 
técnica de trabajo de campo. Las variables son: Peso de los residuos sólidos producidos por la comunidad Untelsina y composición de residuos sólidos. La composición permite conocer qué componentes tienen los residuos, esto permitirá tener un criterio técnico para establecer programas de recuperación y/o reciclaje de residuos. Se utilizaron los siguientes instrumentos: Plano catastral Untels, Ficha para la recolección de datos según tipo de residuo sólido, Ficha para la recolección de datos totales, balanza mecánica, de carga nominal: $15 \mathrm{~kg}$ y exactitud 50 gramos y otros materiales. Se mantuvo estrecha comunicación con la Oficina de Infraestructura.

\section{e. Etapa de Ejecución}

Para el desarrollo de la investigación se consideró el Plan de Seguridad, Salud y Medio Ambiente de acuerdo a las especificaciones de la Normas OHSAS 18001, que persigue la prevención y control de riesgos ocupacionales a través de la participación de todos los participantes en sus respectivas labores diarias, a fin de lograr que ellos mismos sean conscientes de su propia seguridad y la de sus compañeros. El Área de Infraestructura se encargó de la recolección y transporte de los residuos a un área destinada dentro del Campus Universitario depositando los residuos sólidos en el centro de acopio donde se realiza la segregación, como se muestra en la Figura 2. No se consideraron los residuos de los servicios higiénicos, ni los residuos peligrosos del Tópico de Salud y tampoco los del Comedor Universitario, porque a la fecha estaba a cargo de un Concesionario y el personal de limpieza no intervenía dicho espacio. Por la factibilidad de la logística, los parámetros que empleamos para el desarrollo del estudio de caracterización de residuos sólidos son: Generación y composición Se procedió a ordenarlas por áreas generadoras, luego realizamos al pesaje de cada bolsa, como se muestra en la Figura 3. Se tomó registro en la ficha total cada pesaje, posteriormente se procedió a segregar y pesar según tipo de residuo sólido, anotando los datos en la ficha correspondiente. 


\section{f. Etapa de gabinete Sistematización de datos}

Se calcularon los indicadores, la composición. Se utilizaron los intervalos de confianza para estimar las medias poblacionales. Para el procesamiento de los datos se utilizó el software estadístico SPSS versión 23 y la hoja de cálculo MS Excel.

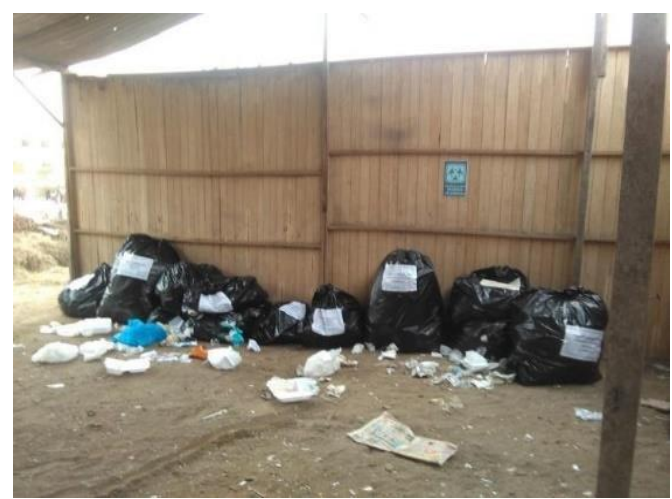

Figura 2: Almacenamiento de los residuos solidos

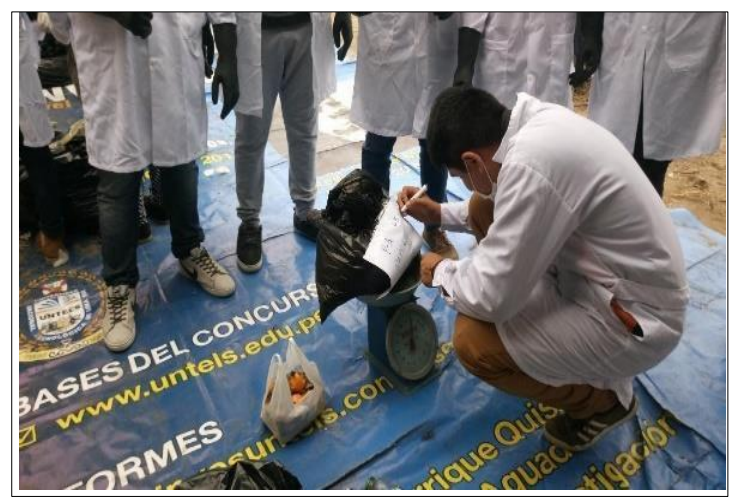

Figura 3: Pesaje y Registro de Datos

\section{Resultados}

\section{a. Generación de residuos}

La generación de residuos sólidos en la UNTELS desagregado por cada una de las diez fuentes de generación (área) se presenta en la Tabla 1 y Figura 4.

Tabla l. Residuos sólidos diario $(\mathrm{Kg})$ según área

\begin{tabular}{|c|c|c|}
\hline \multirow[t]{2}{*}{ Área de Generación } & \multicolumn{2}{|c|}{$\begin{array}{l}\text { Intervalo confianza } \\
\text { de la media }(95 \%)\end{array}$} \\
\hline & Inferior & Superior \\
\hline Rectorado 1 &, 88 & 4,27 \\
\hline $\begin{array}{l}\text { Bibli-Audit. } \\
\text { CEPRE }\end{array}$ & $\begin{array}{l}2,50 \\
6,42\end{array}$ & $\begin{array}{r}5,07 \\
12,10\end{array}$ \\
\hline Dpto. Académico & 5,49 & 7,16 \\
\hline Rectorado 2 & 1,61 & 6,01 \\
\hline Pabellón A & 4,85 & 12,39 \\
\hline Pabellón B & 6,04 & 12,29 \\
\hline Pabellón C & 3,12 & 9,61 \\
\hline Cafetin & 1,29 & 4,1270 \\
\hline $\begin{array}{l}\text { Gimnas-Cent.Méd } \\
\text { Total }\end{array}$ & 2,37 & 8,8109 \\
\hline
\end{tabular}

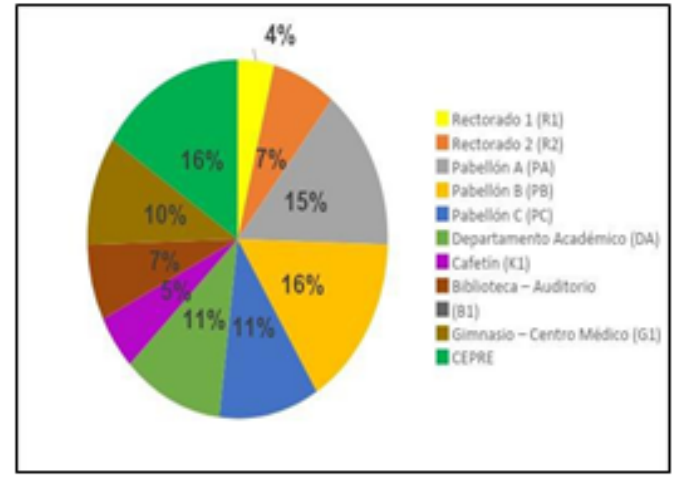

Figura 4. UNTELS: Participación porcentual de generación de residuos sólidos según Area degeneración. 


\section{b. Composición de los residuos sólidos}

En todas las áreas, la generación de residuos electrónicos no resultó significativa. En el área de Biblioteca-Auditorio se evidencia una nula generación de vidrio, en cuanto a papel se genera entre 0,0685 y 0,5886 Kg/día, plástico, entre 0,4255 y 0,7316 Kg/día, materia orgánica 0,4291 y $1,7138 \mathrm{Kg}$ /día y basura propiamente entre 0,8168 y 2,3260 Kg/día, en todos los casos con un nivel de confianza de $95 \%$.

En el área del Centro Pre Universitario los datos evidencian una nula generación de papel y vidrio, respecto al plástico se encuentra entre 1,2529 y 1,7022 kg/día, materia orgánica entre 0,6889 y 2,3947 kg/día y basura propiamente entre 3,60 y 6,62 Kg, con un nivel de confianza de 95\%. En el área del Departamento Académico los datos evidencian una generación de papel entre 0,8260 y 1,2965 kg/día, plástico PET entre 0,2742 y 0,9115, vidrio entre 0,1158 y 0,6026, materia orgánica entre 0,2334 y 1,2074 kg/día y basura propiamente entre 2,7889 y 4,3621 $\mathrm{kg}$ /día, en todos los casos con un nivel de confianza de $95 \%$.

En el área de Gimnasio-Centro Médico los datos evidencian una nula generación de papel y vidrio, en cuanto al plástico PET se genera entre 0,3059 y 1,6941 kg/día, materia orgánica entre 0,6424 y $1,7719 \mathrm{~kg} /$ día y basura propiamente, entre 0,5741 y 5,1402 kg/día. En el área de Cafetín los datos evidencian una nula generación de vidrio y materia orgánica, papel entre 0,0302 y 0,8677 kg/día, plástico PET entre 0,1211 y 1,1810 kg/día, basura entre 0,6145 y 1,6390 $\mathrm{kg}$ /día, todos con un nivel de confianza de $95 \%$.

En el área de Pabellón “A”, los datos evidencian una generación de papel entre 0,1705 y 2,1152 kg/día, plástico PET entre 0,8973 y 2,1242 kg/día, vidrio entre 0,1282 y 0,7832 kg/día, materia orgánica entre 0,7813 y 1,8473 kg/día y basura propiamente entre 0,1651 y 7,5634 kg/día, en todos los casos con un nivel de confianza de 95\%. En el área de Pabellón "B”, los datos evidencian una nula generación de vidrio, papel entre 0,1764 y 0,9093 kg/día, plástico PET entre 0,7006 y $1,5851 \mathrm{~kg} /$ día, materia orgánica entre 0,2316 y 2,2684 kg/día y basura propiamente entre 3,7427 y $5,9858 \mathrm{~kg} /$ día, en todos los casos con un nivel de confianza de $95 \%$. En el área de Pabellón “C” los datos evidencian una nula generación de papel y vidrio, plástico PET entre 0,2104 y 1,5753 kg/día, materia orgánica entre 0,1775 y 2,2368 kg/día y basura propiamente entre 1,7936 y $5,6636 \mathrm{~kg}$ /día, todos con un nivel de confianza de $95 \%$. 
En el área del Rectorado 1, los datos evidencian una nula generación promedio de cualquier tipo de residuos sólidos con un nivel de confianza de 95\%. En el área del Rectorado 2, los datos evidencian una nula generación promedio de vidrio y materia orgánica, papel entre 0,6782 y 2,6218 kg/día, plástico PET entre 0,0976 y 0,7167 kg/día y basura propiamente entre 0,2501 y $2,8213 \mathrm{~kg} /$ día, todos con un nivel de confianza de $95 \%$.

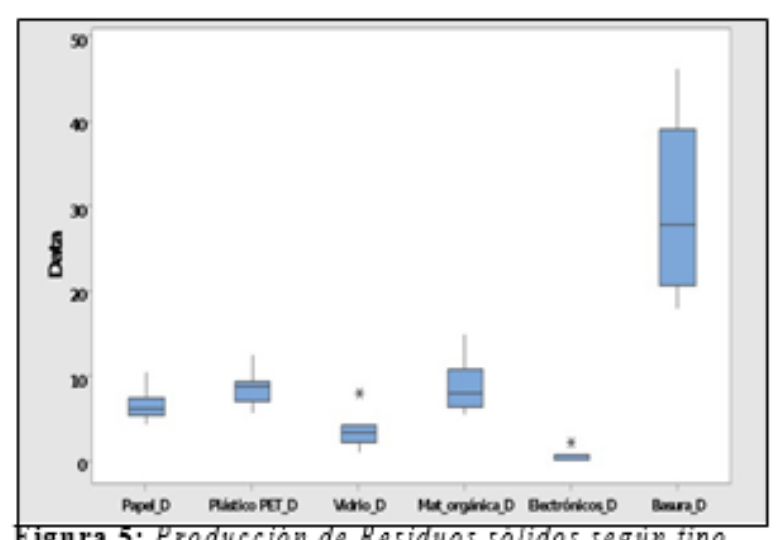

Figura 5: Produccion de Residuos solidos segun tipo (Kg/dia)

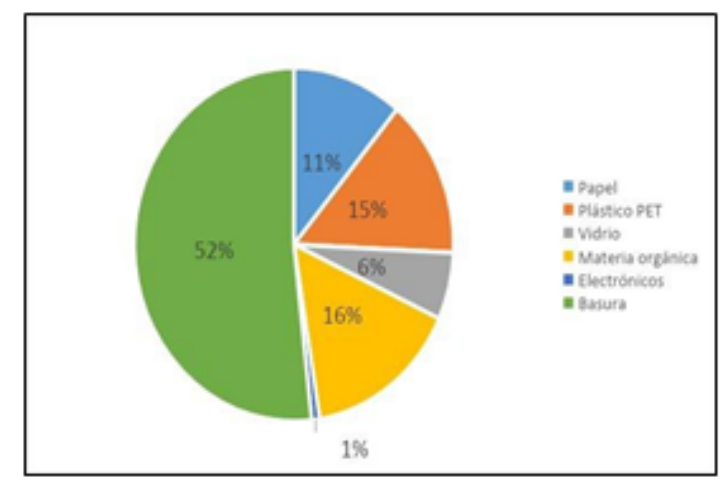

Figura 6: Composición fisica promedio de los residuos sólidos en la UNTELS

Se estima una producción promedio diaria de papel entre $(4,635 ; 8,340) \mathrm{kg}$, la producción de plástico PET oscila entre $(6,547 ; 10,449) \mathrm{kg}$, la producción de vidrio está entre $(1,517 ; 5,657)$ $\mathrm{kg}$, materia orgánica entre $(6,00 ; 12,08) \mathrm{kg}$, residuos electrónicos entre $(-0,233 ; 1,164)$ $\mathrm{kg}$, basura entre $(20,71 ; 39,57) \mathrm{Kg}$ y la producción total diaria de residuos sólidos se encuentra entre $(45,69 ; 70,74) \mathrm{kg}$, con un nivel de confianza del $95 \%$ como se muestra en la Figura 5 y Figura 6.

\section{Discusión}

Se estima que las áreas que generan mayor cantidad promedio de residuos sólidos son: el Centro Pre-Universitario (entre 6,42 y 12,10 Kg por día), el Pabellón “B” (entre 6,04 y 12,30 Kg por día) y el Pabellón “A” (entre 4,85 y 12,40 Kg por día) en todos los casos con un nivel de confianza de $95 \%$, zonas donde hay mayor presencia de estudiantes, por lo que pueden establecerse los puntos más vulnerables en generación de residuos sólidos y la necesidad de disponer de tachos permanentemente, así como a quienes deben estar preferentemente dirigidos las actividades de sensibilización. La generación per cápita (GPC) es de $0.02 \mathrm{Kg}$, valor que comparado con el reporte del (Organismo de Evaluación y Fiscalización Ambiental, 2014) y con el resultado obtenido por (Ramírez, 2014), es bastante inferior y se convierte en muy buen 
indicador. Se determinó que la generación total de residuos sólidos es de $58.22 \mathrm{Kg}$ /día, equivalente a 1.51 ton/mes y 15.14 ton/año, estos resultados son importantes porque, de ser utilizados servirían para la planificación del personal de limpieza y los recursos que se puedan asignar para la recolección de los residuos. En cuanto a la composición, la cantidad de residuos electrónicos no es significativa en ninguna área de generación, lo cual nos evidencia un buen manejo de este tipo de residuo dentro de los laboratorios, la generación de vidrio sólo resultó significativa en el Departamento Académico y en el Pabellón “A”. Se debe tomar en cuenta que el Pabellón "A" está conformado por aulas y algunos laboratorios, por lo que se sugiere analizar el origen del vidrio, la generación de papel resultó significativa en la Biblioteca Auditorio, Departamento Académico, cafetín, Pabellón “A” y “B”, y Rectorado 2. La generación de plástico y de basura fue significativa en todas las áreas de generación, salvo en el Rectorado 1, la materia orgánica fue significativa en todas las áreas de generación salvo el cafetín, Rectorado 1 y Rectorado 2, estos resultados nos llevan a considerar el desarrollo de estrategias como la ubicación de puntos ecológicos y evidencian la necesidad de una cultura de toma de conciencia en el cuidado ambiental. La jerarquización por tipo de residuo sólido permitió encontrar en primer lugar a la basura propiamente (52\%), seguido de materia orgánica (16\%), plástico PET (15\%) papel (11\%), vidrio (6\%) y finalmente electrónicos $(1 \%)$, expresados en porcentajes redondeados. Nuestro resultado concuerda con lo encontrado por (Ipanaque, Lazo, Solar, \& Zeta, 2013) puesto que los residuos reaprovechables que más genera la UNTELS son las botellas de plástico. A diferencia de (Vásquez Estela, Bermejo Sánchez, \& Sarmiento Ramos, 2013). Se encontró que la UNTELS, a pesar de sus actividades propias, no genera papel de manera importante.

Destaca la generación de basura, entre 20.71 y $39.57 \mathrm{Kg}$ diariamente, y la generación de residuos electrónicos no es significativa. En concordancia con el (Comité Ambiental Universitario UNAP, 2018) de la UNAP, podemos decir que la UNTELS tampoco genera residuos peligrosos de laboratorios de manera importante. Con respecto a las pilas, baterías, tóner y cartuchos de tinta de impresoras, los fluorescentes y porta-fluorescentes o cualquier tipo de luminarias también tienen una generación no continua, motivo por el cual durante el periodo de muestreo tampoco se encontró de manera significativa, además de estar categorizados como residuos sólidos peligrosos. 
Contrariamente a lo mencionado por el (Comité Ambiental Universitario UNAP, 2018) de la UNAP en el caso de los residuos sólidos generales o comunes muestran un avance importante puesto que en la UNAP ya segrega en la fuente: aulas, oficinas, etc., luego el personal de servicio o limpieza lo traslada hasta la vía pública para ser recogida por una EPS, emprendimiento que puede ser un modelo a seguir por nuestra casa de estudios.

\section{Conclusiones}

Como resultados globales tenemos que la generación per cápita (GPC) es de $0.02 \mathrm{Kg}$, valor que comparado con otros estudios se convierte en muy buen indicador, dada la ubicación de la Universidad en el distrito de Villa el Salvador. La generación total de residuos sólidos es de $58.22 \mathrm{Kg} /$ día, equivalente a 1.51 ton/mes y 15.14 ton/año. La jerarquización por tipo de residuo sólido arrojó en primer lugar a la basura propiamente (52\%), seguido de materia orgánica (16\%), plástico PET (15\%) papel (11\%), vidrio (6\%) y finalmente electrónicos (1\%), expresados en porcentajes redondeados. A nivel poblacional podemos afirmar con nivel de confianza de $95 \%$ que la generación de basura oscila entre 20.71 y $39.57 \mathrm{Kg}$ diariamente, y la generación de residuos electrónicos no es significativa.

La clasificación de residuos sólidos sirvió para estimar que las áreas que generan mayor cantidad promedio de residuos sólidos son: el Centro Pre-Universitario, el Pabellón “B” y el Pabellón "A". Siendo las botellas de plástico el mayor tipo de residuo reaprovechable y en segundo lugar el papel, los cuales serían oportunidades de producción viable que genere beneficios al plan de gestión de residuos sólidos. Este tipo de residuos principalmente se genera en el Departamento Académico (papel), en el Gimnasio-Centro Médico (plástico PET), en el Cafetín y Rectorado 2 (papel, el plástico PET), en el Pabellón “A” (papel, plástico PET).

\section{Referencias Bibliográficas}

Alves, E., Felipe de Souza, V., \& Rodrigues, D. F. (2019). Estratégia paradestinação de resíduos sólidos recicláveis provenientes de universidades brasileiras. Multitemas, 24(57), 7. https://doi.org/10.20435/multi.v24i57.2106 
Amézquita, R. del P. (2019). Propuesta de un sistema de gestión de manejo de residuos sólidos para minimizar el impacto ambiental en una universidad privada de la Región Lambayeque. Universidad Católica Santo Toribio de Mogrovejo.

Cabañas, E., Díaz, M., y Oliva, M. (2019). Densidad de los residuos sólidos de tres instituciones educativas de la ciudad de Chachapoyas, departamento de Amazonas. Rev.de Investig. Agroproducción $\quad$ Sustentable,3(1), 27. https://doi.org/10.25127/aps.20191.479

Comité Ambiental Universitario (UNAP). (2018). Plan de Manejo de Residuos Sólidos no Peligrosos y Peligrosos Universidad Nacional De La Amazonía Peruana. https://www.unapiquitos.edu.pe/investigacion/UEEA/descargas/IND 19Plan MRS no peligrosos y peligrosos-UNAP-2018-PUBLICAR.pdfEche, K., y Sánchez, R. (2016). Plan de Manejo de Residuos Sólidos del Colegio Avante. (p.

97). $\quad$ http://repositorio.lamolina.edu.pe/bitstream/handle/UNALM/2252/Q70E23- T.pdf? sequence $=1 \&$ isAllowed $=\mathrm{y}$

Ferreira, E., Lobo Vasconcelos, M., Rodrigues, E., \& Ferreira, F. (2017). Gestão da Coleta Seletiva de Resíduos Sólidos no CampusPampulha da UFMG: Desafios e Impactos Sociais. Revista de Gestão Ambiental e Sustentabilidade: GeAS, 6(3), 131-149. https://doi.org/10.5585/geas.v6i3.821

Ministerio del Ambiente. (2015a). Guía metodológica para el desarrollo del Estudio de

Caracterización de Residuos Sólidos Municipales (EC-RSM).

http://sial.segat.gob.pe/documentos/guia-metodologica-desarrollo-estudiocaracterización-residuos-sólidos.

Organismo de Evaluación y Fiscalización Ambiental. (2014). Fiscalización ambiental en residuos sólidos destión municipal provincial. https://www.oefa.gob.pe/?wpfb_dl=13926.

Ramírez, W. (2014). Caracterización de los residuos sólidos de las instituciones educativas urbanas para un manejo adecuado de la basura en el distrito de Barranca, año2021.

Tello, P., Martínez, E., Daza, D., Soulier, M., y Terraza, H. (2010). Informe de la evaluación regional del manejo de residuos sólidos urbanos en AméricaLatina y El Caribe 2010. file://C:/Users/Usuario/Downloads/Informe-de-la-evaluación- regional-del-manejode- residuos-sólidos-urbanos-en-América-Latina-y-el-Caribe- 2010.pdf.

Vásquez, E., Bermejo, D., Sánchez, F., y Sarmiento, J. (2013). Percepción y manejo de residuos sólidos en universitarios.

Universidad Nacional José Faustino Sánchez Carrión (2013). Perception and solid waste management in college. 2013José Faustino Sánchez Carrión National University. 1-10. repositorio.unjfsc.edu.pe/handle/UNJFSC/1626. 